\title{
Meta-analysis of nanoparticle albumin-bound paclitaxel used as neoadjuvant chemotherapy for operable breast cancer based on individual patient data (JBCRG-S01 study)
}

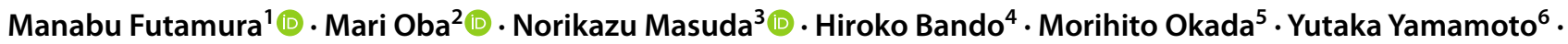 \\ Takanori Kin ${ }^{7} \cdot$ Toshiaki Saeki $^{8}$. Takeshi Nagashima ${ }^{9} \cdot$ Takashi Kuwayama $^{10}$. Uhi Toh ${ }^{11}$ - Akira Hirano ${ }^{12}$. \\ Masafumi Inokuchi ${ }^{13} \cdot$ Kazuhiko Yamagami $^{14} \cdot$ Yutaka Mizuno $^{15} \cdot$ Yasuyuki Kojima ${ }^{16}$ - Takahiro Nakayama ${ }^{17}$. \\ Hiroyuki Yasojima ${ }^{3}$ Shinji Ohno ${ }^{18}$
}

Received: 18 October 2020 / Accepted: 8 March 2021 / Published online: 3 April 2021

(c) The Author(s) 2021

\begin{abstract}
Background Nanoparticle albumin-bound paclitaxel (nab-PTX), a novel taxane formulation, was developed to avoid cremophor/ethanol-associated toxicities including peripheral neuropathy and hypersensitivity. At least 35 phase II studies using combined nab-PTX and anthracycline in neoadjuvant settings are registered in Japan. We analyzed the efficacy and safety of nab-PTX based on patient characteristics in these studies.

Methods We conducted a meta-analysis using individual patient data (IPD) to investigate the average efficacy of nab-PTXcontaining regimens as neoadjuvant chemotherapy for operable breast cancer. IPD were provided by principal investigators who agreed to participate. The primary endpoint was pathological complete response (pCR) rate of each breast cancer subtype.

Results We analyzed the data of 16 studies involving 753 patients. The overall crude frequencies of pCR (ypT0 ypN0, ypT0/ is ypN0, and ypT0/is ypNX) were 18.1, 26.0, and 28.6\%, respectively. Specifically, the frequencies were $6.7,10.2$, and $13.4 \%$ for luminal $(n=343)$; 40.5, 63.5, and 68.9\% for human epidermal growth factor receptor $2($ HER2)-rich, $(n=74)$; 21.9, 40.6, and $42.7 \%$ for luminal/HER2 $(n=96)$; and 26.3, 31.5, and 32.3\% for triple-negative breast cancers (TNBC) $(n=232)$. The multivariate analyses indicated that HER2 positivity, TNBC, high Ki-67, high nuclear grade, and weekly nab-PTX administration were significantly associated with the pCR. The proportion of hematological toxicities (neutropenia (39.7\%) and leukopenia (22.5\%)), peripheral sensory neuropathy (9.7\%), myalgia (5.7\%), and arthralgia (4.7\%) was higher than grade 3 adverse events, but most patients recovered.

Conclusions Nab-PTX is a safe and acceptable chemotherapeutic agent in neoadjuvant settings, particularly for aggressive cancers. UMIN-CTR\#: UMIN000028774
\end{abstract}

Keywords Nanoparticle albumin-bound paclitaxel $\cdot$ Meta-analysis $\cdot$ Individual patient data $\cdot$ Pathological complete response $\cdot$ HER2-rich

\section{Introduction}

Although taxane is a current gold standard chemotherapeutic agent for breast cancer (BC), adverse events (AEs) such as peripheral neuropathy and hypersensitivity are often problematic for patients. Nanoparticle albumin-bound

Manabu Futamura

mfutamur@gifu-u.ac.jp

Extended author information available on the last page of the article paclitaxel (Nab-PTX) is a novel taxane formulation that was developed to avoid the toxicities associated with cremophor/ethanol co-solvents, such as the aforementioned peripheral neuropathy and hypersensitivity reactions [1, 2]. Nab-PTX showed higher tumor suppression in a mouse model than conventional paclitaxel used at a high concentration [3]. As taxol plays an important role in BC therapy, nab-PTX was first utilized for metastatic BC, resulting in longer progression-free survival (PFS) than that achieved with either paclitaxel or docetaxel $[4,5]$. Nab-PTX has been reported to induce specific AEs such as arthralgia, 
myalgia, and peripheral neuropathy, but they are transient and controllable [5]. These reports suggest wide applications of nab-PTX for BC therapy. However, a few largescale phase III studies using a nab-PTX-containing regimen in a neoadjuvant setting have reported remarkable findings that nab-PTX is more effective than paclitaxel [6].

In Japan, nab-PTX was approved for use and insurance coverage in July 2010; it has been widely used in both neoadjuvant and metastatic settings. At least 35 phase II neoadjuvant studies using nab-PTX have been registered in the University Hospital Medical Information NetworkClinical Trial Registry (UMIN-CTR) [7]. Of these, several studies using a combination of nab-PTX and anthracycline as neoadjuvant chemotherapy (NAC) have been reported [8-13]. However, these studies have not progressed to phase III randomized controlled trials (RCTs) and their sample sizes have been small. In addition, the results varied because the distribution of patient characteristics differed among the studies. Thus, to estimate the efficacy and safety of nab-PTX precisely in a neoadjuvant setting, we aimed to extract individual patient data (IPD) from studies on nab-PTX-containing regimens registered in UMINCTR and analyze the efficacy and safety of nab-PTX based on patient characteristics in a meta-analysis [14].

\section{Materials and methods}

\section{Specific criteria}

This study is a collaborative meta-analysis of phase II trials using IPD to summarize published and unpublished evidence on the efficacy of nab-PTX-containing regimens. Patients with operable BC (cStages I-III) who received NAC with nab-PTX were included. The primary endpoint was the pathological complete response (pCR) rate in each subtype. Clinical subtypes were defined by immunohistochemical evaluation according to the General Rules for Clinical and Pathological Recording of Breast Cancer (17th edition) based on the UICC-TNM classification [15]. The three definitions of pCR were as follows: (1) ypT0 ypN0, no invasive or noninvasive residual in the breast and lymph nodes; (2) ypT0/is ypN0, no invasive residual in the breast and lymph nodes; and (3) ypT0/is ypNX, no invasive residual in the breast [16]. The secondary endpoints were frequency of greater than Grade 3 AEs ( $\geq$ G3), total dose of nab-PTX (mg/body), disease-free survival (DFS), and overall survival (OS). DFS was defined as the time to relapse or all-cause death from the date of trial registration. OS was defined as the time to all-cause death from the date of registration. This study is registered at UMINCTR under UMIN000028774.

\section{Search strategy and eligibility criteria}

The inclusion criteria were as follows: (1) phase II clinical trial(s) started after July 2010, (2) principal investigators (PIs) agreed to provide IPD, (3) Nab-PTX-containing regimens were used for NAC in chemo-naïve, operable $\mathrm{BC}$ patients, (4) registered at UMIN-CTR, (5) approved by an ethics committee, (6) more than 10 patients were enrolled, and (7) clinical study had already been completed (unpublished data were available). Our inclusion criteria for safety analysis using IPD were (1) operable (Stages I-III), (2) patients with no previous treatment, and (3) patients who underwent at least one cycle of each regimen. Our inclusion criteria for efficacy analysis were (1) patients who underwent surgery and (2) progressive disease (PD).

\section{Data collection}

Data collection from the clinical trials was approved by each ethical committee and consent was obtained from the sponsor if necessary. All the studies provided patients with an opportunity to opt-out before data submission. Data pertaining to the following variables were requested from all studies: age, menopause, histology of pre/post NAC [estrogen receptor (ER), progesterone receptor (PgR), HER2, Ki-67, nuclear grade (NG), and histological grade (HG)], regimen, doses of NAC agents, surgical methods, image evaluation, $A E s \geq G 3$, effect of NAC, DFS, and OS.

\section{Assessment of studies}

Before analysis, we checked the risk of bias using the Risk of Bias Assessment Tool for Nonrandomized Studies (RoBANS) and Cochrane training [17, 18]. Next, we constructed a forest plot and evaluated the heterogeneity of the pCR rates among the studies. The $I^{2}$ statistic, which is the ratio of heterogeneity to total variance in the $\mathrm{pCR}$ rates among all studies, was calculated. A funnel plot was constructed to assess publication bias, which displayed the relationship between the study size and effect size.

\section{Statistical analysis using IPD}

The pCR rate and $95 \%$ confidence interval (CI) were calculated for each study, for all patients, and for subgroups. The preplanned subgroup variables were menopause, age, NG, Ki-67, clinical stage, use of nab-PTX, clinical response, subtype, and HER2 status. Forest plots were used to display the $\mathrm{pCR}$ rates by subgroups. The association of clinical variables with the achievement of $\mathrm{pCR}$ was assessed using univariate and multivariable logistic models and expressed 
as odds ratios (ORs). A multiple imputation approach was used to manage missing clinical variables in the multivariate logistic model. The proportion of $\mathrm{AE} \geq \mathrm{G} 3$ and average total dose of nab-PTX per patient were also calculated. The difference in the proportion of AEs between q3w (every 3 weeks) and weekly nab-PTX was tested using the Chi-square test. The total dose of nab-PTX administered per patient was compared between nab-PTX regimens using $t$ tests. DFS and OS were summarized using the Kaplan-Meier method and compared using the log-rank test. Hazard ratios (HRs) were calculated using a crude Cox proportional hazards model. All statistical tests were two-sided, and results with $p<0.05$ were considered statistically significant. Statistical multiplicity was not adjusted. All statistical analyses were performed using SAS software version 9.4 (SAS Institute, Cary, NC) and R package "metaphor."

\section{Results}

\section{Characteristics of clinical trials}

We found 35 studies in the UMIN-CTR, which were reviewed using the PRISMA IPD flow diagram shown in Fig. 1a [19]. Twelve studies were either incomplete or ongoing, and one was a duplicate; six studies failed to provide IPD. Thus, the datasets of 16 studies (6 published [8-13] and 10 unpublished) involving 758 patients were selected for further analysis. All included studies were phase II; 15 were single-arm and one was an RCT. The protocol regimens utilized both nab-PTX and anthracycline. Nab-PTX was administered either q3w (11 studies) or weekly (5 studies). Thirteen studies administered nab-PTX followed by anthracycline, and three studies administered anthracycline followed by nab-PTX (Table 1). For safety evaluation, 753 patients were analyzed because three patients did not receive treatment, and two metastatic cases were excluded. For efficacy evaluation, 745 patients were analyzed because four patients who denied surgery or treatment and four patients who did not visit the hospital were excluded (Fig. 1b).

We were provided all study protocols and IPD data (with a few missing data points) by each principal investigator; the data were subjected to a quality check using RoBANS (Suppl. Fig. 1). Eventually, the risk of bias for the metaanalysis of non-RCTs was deemed to be moderate. The $\mathrm{I}^{2}$ statistic (68.8\%) indicated a moderate heterogeneity among the 16 studies (Suppl. Fig. 2a). The funnel plot showed that most of the studies were distributed symmetrically, except for two small studies that reported low pCR rates (Suppl. Fig. 2b). Table 1 shows the characteristics of the clinical trials. The two studies that reported the low pCR rates included only luminal or triple-negative subtypes, and higher proportions of cStage III than the other studies. The distribution of patient characteristics varied among the trials.

\section{Characteristics of the patients}

The characteristics of the patients are summarized in Table 2. All HER2-positive cases, except four (including luminal/HER2 cases), were treated by the combination of nab-PTX and trastuzumab. Of the 758 patients (mean age 52.2 years), the number of patients with cStages I, IIA, IIB, IIIA, IIIB, and IIIC was 47 (6.2\%), 260 (34.5\%), 279 (37.1\%), $88(11.7 \%), 35$ (4.6\%), and $44(5.8 \%)$, respectively. Luminal, HER2-rich, luminal/HER2, and TNBC subtypes were observed in 347 (46.1\%), 75 (10.0\%), 96 (12.7\%), and $235(31.2 \%)$ patients, respectively. Ki-67, NG, and HG were not routinely evaluated in some clinical studies. Most HER2rich populations involved high-NG, high Ki-67 ( $\geq 40 \%$; median value was $40 \%$ ), and the use of trastuzumab. The patient characteristics after NAC are shown in Suppl. [20, 21].

\section{PCR rates based on IPD}

Among the efficacy analysis population (745 patients), surgery was performed in 743 patients. In each subtype, the three pCRs (ypT0 ypN0, ypT0/is ypN0, and ypT0/is ypNX) were observed in $6.7 \%$ (95\% CI: 4.3-9.9), 10.2\% (7.2-13.9), and $13.4 \%(10.0-17.5)$ of the patients for luminal; $40.5 \%$ (29.3-52,6), 63.5\% (51.5-74.4), and 68.9\% (57.1-79.2) of the patients for HER2-rich; $21.9 \%$ (14.1-31.5), $40.6 \%$ (30.7-51.1), and $42.7 \%(32.7-53.2)$ of the patients for luminal/HER2; and 26.3\% (20.7-32.5), 31.5\% (25.5-37.9), and $32.3 \%$ (26.4-38.8) of the patients for TNBC, respectively (Fig. 2). A forest plot of the proportion of ypT0 ypN0, ypT0/is ypN0, and ypT0/is ypNX patients according to subgroup variables is shown in Suppl. Fig. 3. In Suppl. Fig. $3 b$ for the population of ypT0/is ypN0, the pCR rates for NG 1, 2, and 3 were $7.7 \%$ (95\% CI: 3.9-13.3), 17.8\% (11.7-25.3), and 34.6\% (29.3-40.3); those for low $(<40 \%)$ and high $(\geq 40 \%) \mathrm{Ki}-67$ expression were $12.5 \%(8.8-17.2)$ and $33.9 \%$ (28.6-39.5); those for cStages I, IIA, IIB, and IIIA were $37.0 \%$ (23.2-52.5), 31.4\% (25.8-37.4), $23.0 \%$ (18.2-28.4), and 23.5\% (15.0-34.0); and those for HER2positive and -negative cases were $50.6 \%(42.8-58.3)$ and $18.8 \%$ (15.7-22.2), respectively. The other forest plots for ypT0 ypN0 and ypT0/is ypNX revealed results similar to those shown in Suppl. Fig. 3a, c.

\section{Clinical variables associated with pCR}

The ORs of clinical variables are shown in Table 3. The univariate analysis showed that subtype, NG, HG, high Ki-67, early cStage, use of trastuzumab, and effect of NAC 
Fig. 1 PRISMA flow diagrams for the meta-analysis. a Identification of studies. b Inclusion/ exclusion of patients a

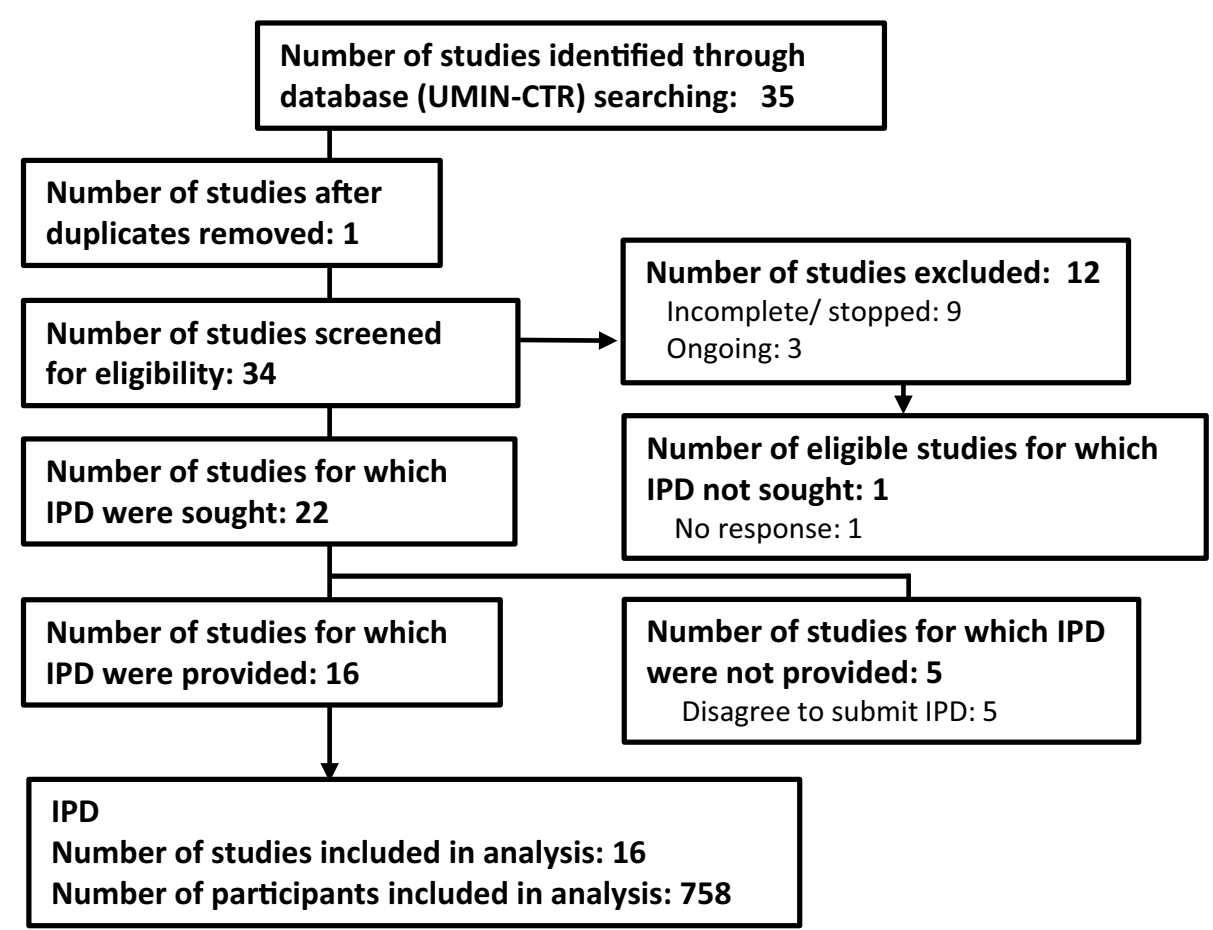

b

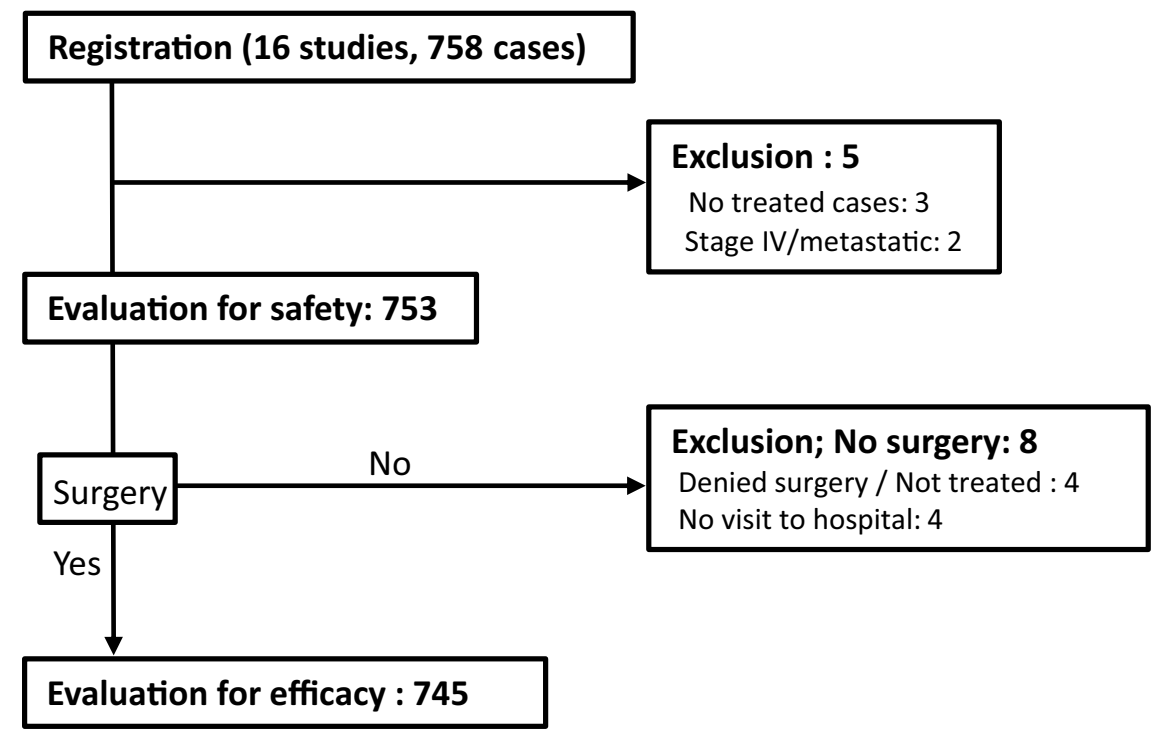

were significantly associated with each pCR rate. Menopause, HG, and the use of trastuzumab were not assessed using the multivariate model because of the collinearity between menopause and age, as well as HG, NG, HER2positive status, and the use of trastuzumab. Clinical evaluation of NAC was not performed because it was a result of, rather than a risk factor for, the response. The ORs for the HER2-rich, luminal/HER2, and TNBC groups were 15.14
(7.81-29.33), 6.33 (3.50-11.45), and 2.97 (1.84-4.80), respectively. Those for NG 2 and 3 were $1.72(0.79-3.73)$ and 2.90 (1.48-5.71) times higher than those for NG 1, and high $\mathrm{Ki}-67$ had 2.51 (1.52-4.15) times higher ORs than low Ki-67. The OR for weekly nab-PTX was 2.14 (1.03-4.43) times higher than that for q3w nab-PTX. The ORs for ypT0 ypN0 and ypT0/is ypNX were similar to that for ypT0/is ypN0 (Suppl. Table 2a, b). 


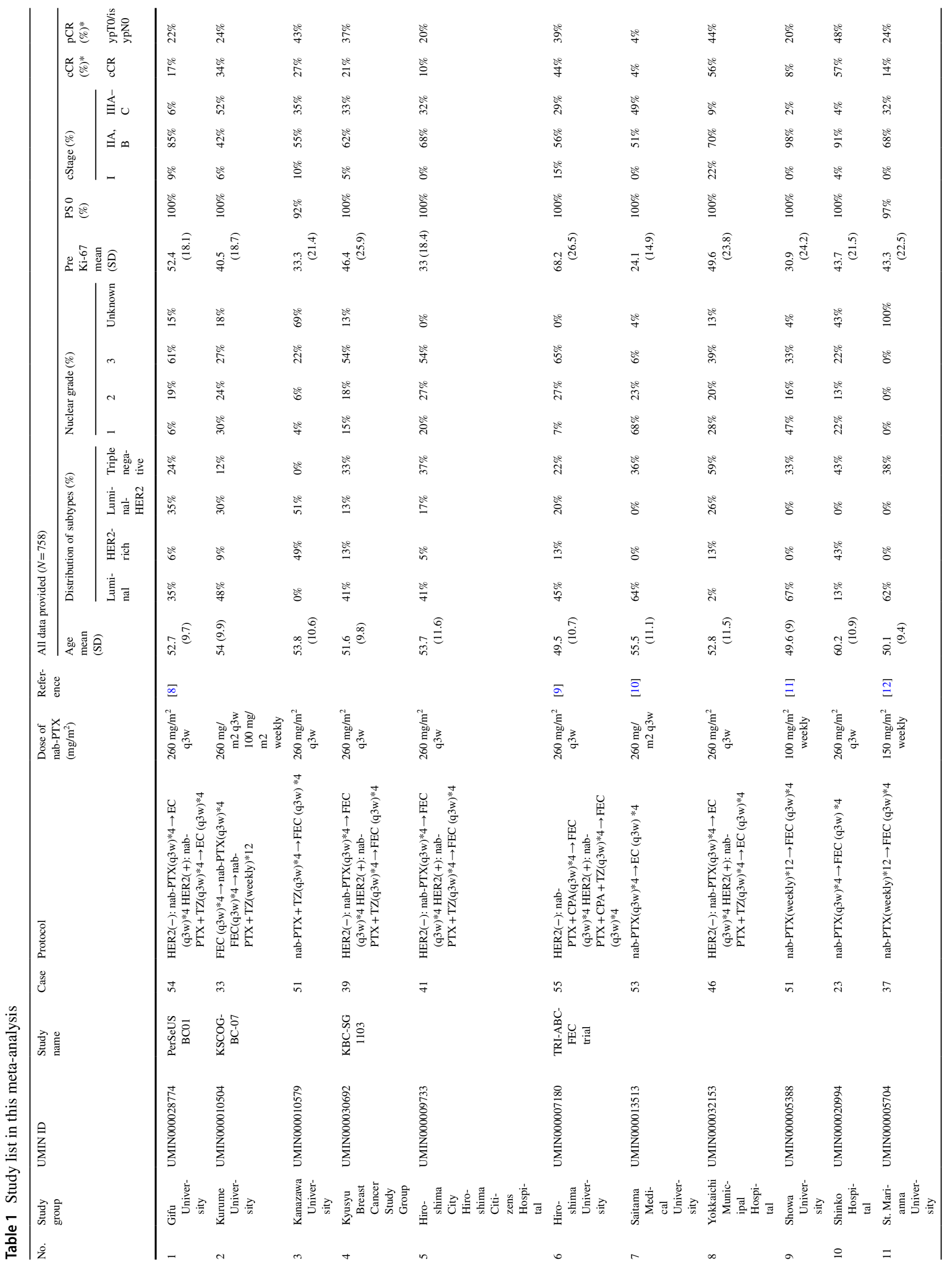




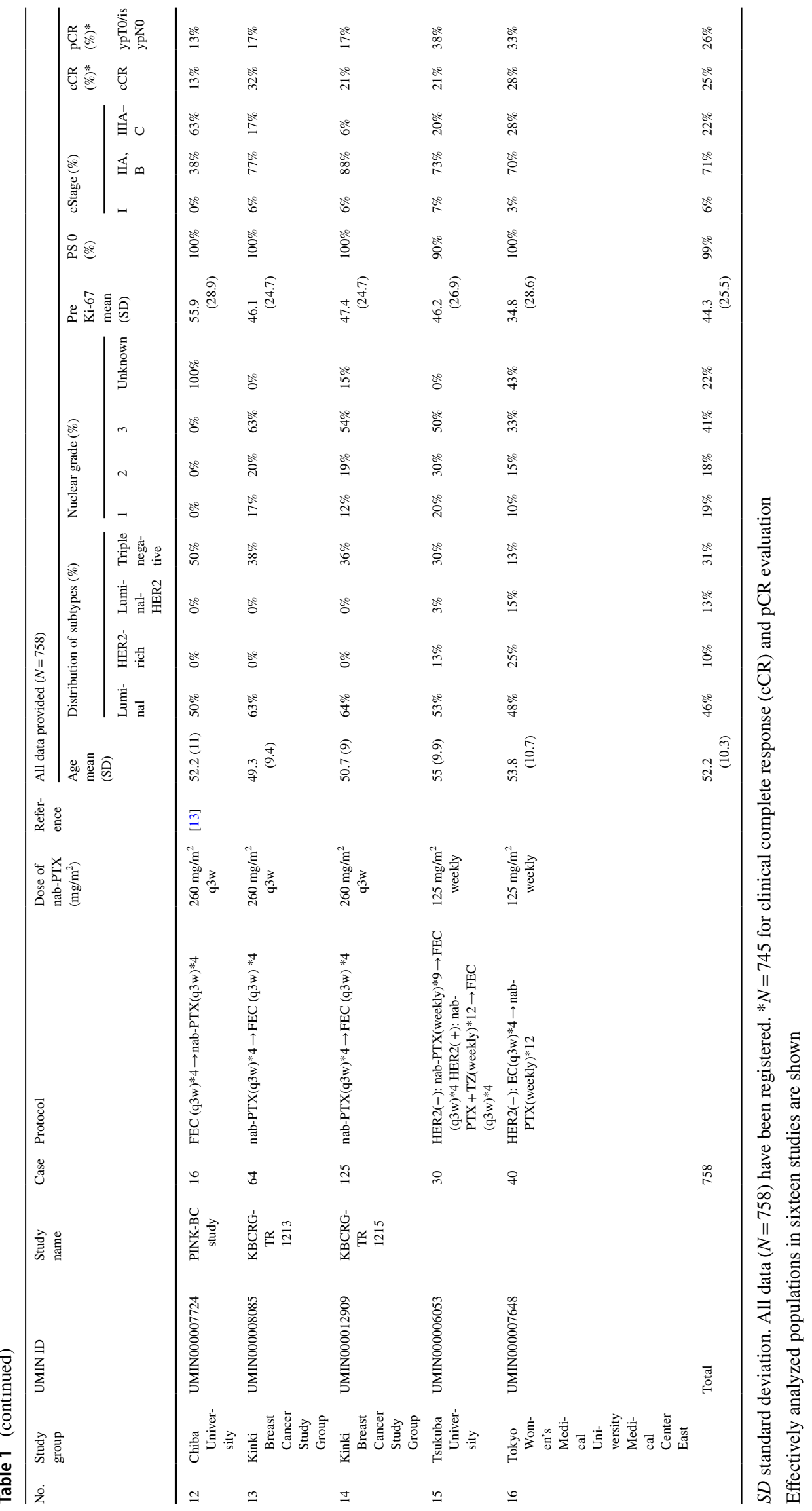


Table 2 Patient characteristics for evaluation of the total population and each subtype

\begin{tabular}{|c|c|c|c|c|c|c|}
\hline & & Cases $(\%)$ & Luminal (\%) & HER2-rich (\%) & Luminal/HER2 (\%) & Triple negative (\%) \\
\hline Sex & Female & $753(100.0 \%)$ & $347(100 \%)$ & $75(100 \%)$ & $96(100 \%)$ & $235(100 \%)$ \\
\hline \multirow[t]{5}{*}{ Age } & mean (SD) & $52.2(10.3)$ & $51.5(9.9)$ & $54.9(10.0)$ & $51.6(10.6)$ & $52.7(10.7)$ \\
\hline & $<40$ & $102(13.6 \%)$ & $47(13.5 \%)$ & $8(10.8 \%)$ & $15(15.6 \%)$ & $32(13.6 \%)$ \\
\hline & $40-59$ & $439(58.4 \%)$ & $211(60.8 \%)$ & $43(58.1 \%)$ & $55(57.3 \%)$ & $130(55.3 \%)$ \\
\hline & $60 \leq$ & $211(28.1 \%)$ & $89(25.6 \%)$ & $23(31.1 \%)$ & $26(27.1 \%)$ & $73(31.1 \%)$ \\
\hline & UK & 1 & 0 & 1 & 0 & 0 \\
\hline \multirow[t]{3}{*}{ Menstruation } & Premenopausal & $362(48.2 \%)$ & $188(54.2 \%)$ & $24(32.4 \%)$ & $41(43.2 \%)$ & $109(46.4 \%)$ \\
\hline & Postmenopausal & $389(51.8 \%)$ & $159(45.8 \%)$ & $50(67.6 \%)$ & $54(56.8 \%)$ & $126(53.6 \%)$ \\
\hline & UK & 2 & 0 & 1 & 1 & 0 \\
\hline \multirow[t]{3}{*}{ Performance status } & 0 & $745(99.2 \%)$ & $345(99.4 \%)$ & $73(98.6 \%)$ & $94(98.9 \%)$ & $233(99.1 \%)$ \\
\hline & 1 & $6(0.8 \%)$ & $2(0.6 \%)$ & $1(1.4 \%)$ & $1(1.1 \%)$ & $2(0.9 \%)$ \\
\hline & UK & 2 & 0 & 1 & 1 & 0 \\
\hline \multirow[t]{6}{*}{ Histology } & IDC (tuble-forming type) & $88(11.8 \%)$ & $39(11.3 \%)$ & $8(11 \%)$ & $23(24 \%)$ & $18(7.7 \%)$ \\
\hline & IDC (solid type) & $179(23.9 \%)$ & $65(18.8 \%)$ & $19(26 \%)$ & $13(13.5 \%)$ & $82(35.2 \%)$ \\
\hline & IDC (scirrhous type) & $321(42.9 \%)$ & $164(47.4 \%)$ & $33(45.2 \%)$ & $46(47.9 \%)$ & $78(33.5 \%)$ \\
\hline & IDC (special type) & $32(4.3 \%)$ & $17(4.9 \%)$ & $2(2.7 \%)$ & $4(4.2 \%)$ & $9(3.9 \%)$ \\
\hline & IDC (UK) & $128(17.1 \%)$ & $61(17.6 \%)$ & $11(15.1 \%)$ & $10(10.4 \%)$ & $46(19.7 \%)$ \\
\hline & UK & 5 & 1 & 2 & 0 & 2 \\
\hline \multirow[t]{2}{*}{ ER } & Positive & $430(57.1 \%)$ & $338(97.4 \%)$ & 0 & $92(95.8 \%)$ & 0 \\
\hline & Negative & $323(42.9 \%)$ & $9(2.6 \%)$ & $75(100 \%)$ & $4(4.2 \%)$ & $235(100 \%)$ \\
\hline \multirow[t]{3}{*}{ PgR } & Positive & $336(44.7 \%)$ & $273(78.9 \%)$ & 0 & $63(65.6 \%)$ & 0 \\
\hline & Negative & $416(55.3 \%)$ & $73(21.1 \%)$ & $75(100 \%)$ & $33(34.4 \%)$ & $235(100 \%)$ \\
\hline & UK & 1 & 1 & 0 & 0 & 0 \\
\hline \multirow[t]{2}{*}{ HER 2} & Positive & $171(22.7 \%)$ & 0 & $75(100 \%)$ & $96(100 \%)$ & 0 \\
\hline & Negative & $582(77.3 \%)$ & $347(100 \%)$ & 0 & 0 & $235(100 \%)$ \\
\hline \multirow[t]{4}{*}{ Nuclear grade } & 1 & $144(24.4 \%)$ & $92(32.9 \%)$ & $2(4.5 \%)$ & $11(15.7 \%)$ & $39(19.9 \%)$ \\
\hline & 2 & $137(23.2 \%)$ & $80(28.6 \%)$ & $9(20.5 \%)$ & $22(31.4 \%)$ & $26(13.3 \%)$ \\
\hline & 3 & $309(52.4 \%)$ & $108(38.6 \%)$ & $33(75 \%)$ & $37(52.9 \%)$ & $131(66.8 \%)$ \\
\hline & UK & 163 & 67 & 31 & 26 & 39 \\
\hline \multirow[t]{4}{*}{ Histological grade } & 1 & $55(18.5 \%)$ & $39(22.8 \%)$ & $1(8.3 \%)$ & $1(25 \%)$ & $14(12.7 \%)$ \\
\hline & 2 & $109(36.7 \%)$ & $77(45 \%)$ & $3(25 \%)$ & $2(50 \%)$ & $27(24.5 \%)$ \\
\hline & 3 & $133(4.48 \%)$ & $55(32.2 \%)$ & $8(66.7 \%)$ & $1(25 \%)$ & $69(62.7 \%)$ \\
\hline & UK & 456 & 176 & 63 & 92 & 125 \\
\hline \multirow[t]{3}{*}{ Ki-67 } & $<40 \%$ & $264(46.0 \%)$ & $164(58.4 \%)$ & $17(42.5 \%)$ & $24(42.9 \%)$ & $59(29.9 \%)$ \\
\hline & $\geq 40 \%$ & $310(54.0 \%)$ & $117(41.6 \%)$ & $23(57.5 \%)$ & $32(57.1 \%)$ & $138(70.1 \%)$ \\
\hline & UK & 179 & 66 & 35 & 40 & 38 \\
\hline \multirow[t]{6}{*}{ Stage } & I & $47(6.2 \%)$ & $8(2.3 \%)$ & $6(8 \%)$ & $12(12.5 \%)$ & $21(8.9 \%)$ \\
\hline & IIA & $260(34.5 \%)$ & $115(33.1 \%)$ & $16(21.3 \%)$ & $23(24 \%)$ & $106(45.1 \%)$ \\
\hline & IIB & $279(37.1 \%)$ & $146(42.1 \%)$ & $28(37.3 \%)$ & $34(35.4 \%)$ & $71(30.2 \%)$ \\
\hline & IIIA & $88(11.7 \%)$ & $43(12.4 \%)$ & $15(20 \%)$ & $12(12.5 \%)$ & $18(7.7 \%)$ \\
\hline & IIIB & $35(4.6 \%)$ & $19(5.5 \%)$ & $3(4 \%)$ & $7(7.3 \%)$ & $6(2.6 \%)$ \\
\hline & IIIC & $44(5.8 \%)$ & $16(4.6 \%)$ & $7(9.3 \%)$ & $8(8.3 \%)$ & $13(5.5 \%)$ \\
\hline \multirow[t]{3}{*}{ Use of Trastuzumab } & Yes & $167(22.2 \%)$ & $1(0.3 \%)$ & $71(95.9 \%)$ & $95(99 \%)$ & 0 \\
\hline & No & $584(77.7 \%)$ & $345(99.7 \%)$ & $3(4.1 \%)$ & $1(1 \%)$ & $235(100 \%)$ \\
\hline & UK & 2 & 1 & 1 & 0 & 0 \\
\hline Total & & 753 & & & & \\
\hline
\end{tabular}

$S D$ standard deviation, $U K$ unknown 
Fig. 2 pCR rates in each breast cancer subtype. a ypT0 ypN0, b ypT0/is ypN0, с ypT0/is ypNX. The actual percentage is given above the corresponding bar a

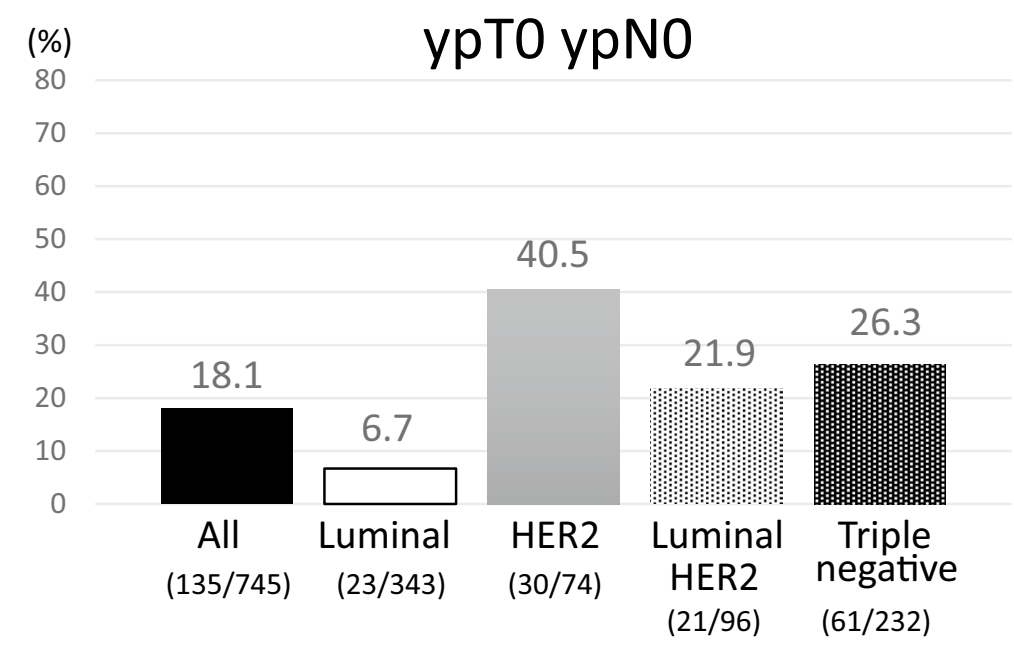

b

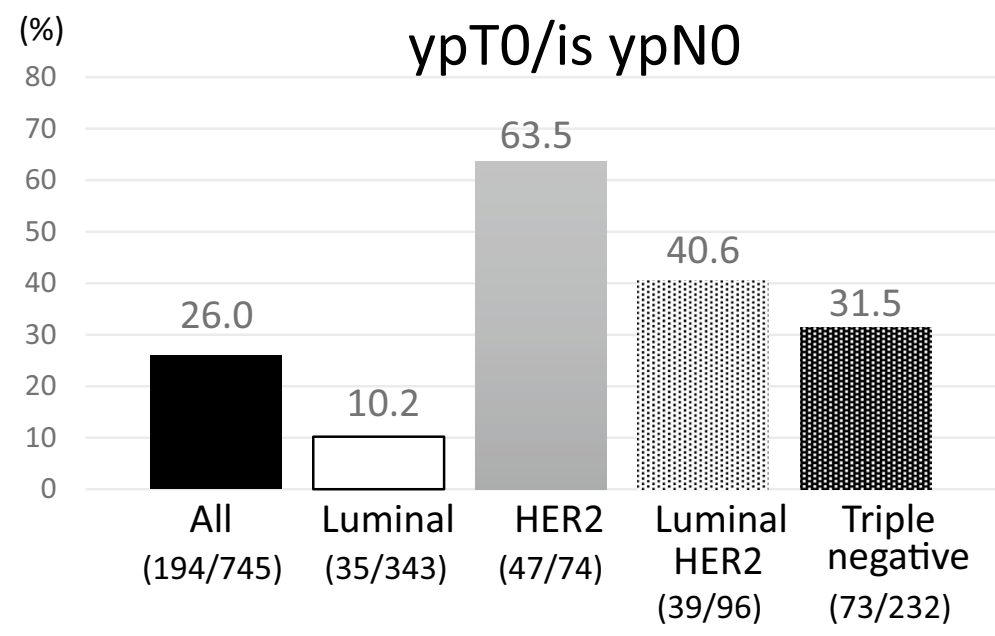


Table 3 Univariate and multivariate logistic regression analyses of pCR (ypT0is ypN0)

\begin{tabular}{|c|c|c|c|c|c|c|c|c|c|}
\hline \multirow{3}{*}{$\begin{array}{l}\text { Variables } \\
\text { Subtype }\end{array}$} & \multirow{3}{*}{$\begin{array}{l}\text { Subgroup } \\
\text { Luminal }\end{array}$} & \multicolumn{4}{|c|}{ Univariable logistic model } & \multicolumn{4}{|c|}{ Multivariable logistic model } \\
\hline & & OR & $95 \% \mathrm{C}$ & & $P$ value & OR & $95 \%$ & & $P$ value \\
\hline & & \multicolumn{8}{|l|}{ Reference } \\
\hline & HER2-rich & 15.32 & 8.50 & 27.59 & $<0.001$ & 15.14 & 7.81 & 29.33 & $<0.001$ \\
\hline & Luminal/HER2 & 6.02 & 3.52 & 10.30 & $<0.001$ & 6.33 & 3.5 & 11.45 & $<0.001$ \\
\hline & Triple negative & 4.04 & 2.59 & 6.31 & $<0.001$ & 2.97 & 1.84 & 4.80 & $<0.001$ \\
\hline Menopause & Post/pre & 1.15 & 0.83 & 1.60 & 0.404 & & & & \\
\hline \multirow[t]{3}{*}{ Age } & $<40$ & Reference & & & & & & & \\
\hline & $40-<60$ & 1.16 & 0.71 & 1.92 & 0.551 & 1.57 & 0.88 & 2.77 & 0.124 \\
\hline & $60-$ & 0.98 & 0.56 & 1.69 & 0.928 & 1.33 & 0.7 & 2.53 & 0.382 \\
\hline \multirow[t]{3}{*}{ Nuclear grade } & 1 & Reference & & & & & & & \\
\hline & 2 & 2.60 & 1.22 & 5.53 & 0.014 & 1.72 & 0.79 & 3.73 & 0.172 \\
\hline & 3 & 6.36 & 3.29 & 12.29 & $<0.001$ & 2.90 & 1.48 & 5.71 & 0.002 \\
\hline \multirow[t]{3}{*}{ Histological grade } & 1 & Reference & & & & - & & & \\
\hline & 2 & 7.47 & 0.95 & 58.67 & 0.056 & - & & & \\
\hline & 3 & 25.20 & 3.37 & 188.37 & 0.002 & - & & & \\
\hline Ki-67 & $40 \% \leq /<40 \%$ & 3.57 & 2.31 & 5.52 & $<0.001$ & 2.51 & 1.52 & 4.15 & $<0.001$ \\
\hline \multirow[t]{3}{*}{ cStage } & I & Reference & & & & Reference & & & \\
\hline & II & 0.63 & 0.34 & 1.19 & 0.153 & 0.83 & 0.41 & 1.71 & 0.618 \\
\hline & III & 0.42 & 0.20 & 0.85 & 0.016 & 0.47 & 0.21 & 1.06 & 0.067 \\
\hline Order of administering nab-PTX & After/Before A & 1.40 & 0.88 & 2.22 & 0.156 & 0.76 & 0.32 & 1.78 & 0.522 \\
\hline Sequence of administering nab-PTX & Weekly/q3w & 1.17 & 0.80 & 1.71 & 0.418 & 2.14 & 1.03 & 4.43 & 0.041 \\
\hline Use of Trastuzumab & & 4.67 & 3.23 & 6.75 & $<0.001$ & - & & & \\
\hline \multirow[t]{4}{*}{ Clinical evaluation of NAC } & $\mathrm{cCR}$ & Reference & & & & & & & \\
\hline & PR & 0.08 & 0.05 & 0.11 & $<0.001$ & & & & \\
\hline & SD & 0.02 & 0.01 & 0.06 & $<0.001$ & & & & \\
\hline & $\mathrm{PD}$ & 0.02 & 0.00 & 0.11 & $<0.001$ & & & & \\
\hline
\end{tabular}

$c C R$ clinical complete response, $P R$ partial response, $S D$ stable disease, $P D$ progressive disease After/Before A After/Before anthracycline

Table 4 Adverse events ( $\geq$ grade 3 ) depending on therapy schedule

\begin{tabular}{|c|c|c|c|c|c|c|c|}
\hline \multirow[t]{3}{*}{ Adverse event } & \multicolumn{6}{|c|}{ Incidence } & \multirow{3}{*}{$\begin{array}{l}\text { Chi-squre test } \\
p \text { value }\end{array}$} \\
\hline & \multicolumn{2}{|l|}{ Total } & \multicolumn{2}{|l|}{$\mathrm{q} 3 \mathrm{w}$} & \multicolumn{2}{|l|}{ Weekly } & \\
\hline & Number & $\%$ & Number & $\%$ & Number & $\%$ & \\
\hline Neutropenia & $298 / 751$ & 39.7 & $115 / 578$ & 19.9 & $83 / 173$ & 48.0 & $<0.0001$ \\
\hline Leukopenia & $169 / 750$ & 22.5 & $107 / 577$ & 18.5 & $62 / 173$ & 35.8 & $<0.0001$ \\
\hline Peripheral sensory neuropathy & $73 / 751$ & 9.7 & $42 / 578$ & 7.3 & $31 / 173$ & 17.9 & $<0.0001$ \\
\hline Febrile neutropenia & $72 / 751$ & 9.6 & $61 / 578$ & 10.6 & $11 / 173$ & 6.4 & $p=0.1$ \\
\hline Myalgia & $43 / 751$ & 5.7 & $19 / 578$ & 3.3 & $24 / 171$ & 14.0 & $<0.0001$ \\
\hline Hepatobiliary disorders & $41 / 751$ & 5.5 & $37 / 578$ & 6.4 & $4 / 173$ & 2.3 & 0.0378 \\
\hline Arthralgia & $36 / 749$ & 4.8 & $13 / 578$ & 2.3 & $23 / 171$ & 13.5 & $<0.0001$ \\
\hline Vomitng & $31 / 751$ & 4.1 & $18 / 578$ & 3.1 & $13 / 173$ & 7.5 & 0.0304 \\
\hline Peripheral motor neuropathy & $17 / 751$ & 2.3 & $16 / 578$ & 2.9 & $1 / 173$ & 0.1 & 0.0893 \\
\hline Infusion reaction & $6 / 751$ & 0.8 & $4 / 578$ & 0.7 & $2 / 173$ & 1.2 & 0.5475 \\
\hline Cardiac disorders & $4 / 751$ & 0.5 & $2 / 578$ & 0.4 & $2 / 173$ & 1.2 & 0.1991 \\
\hline Death & $1 / 753$ & 0.1 & $1 / 580$ & 0.2 & $0 / 173$ & 0.0 & NA \\
\hline
\end{tabular}




\section{Toxicity profiling}

The AEs $\geq$ G3 were as follows: neutropenia, 39.7\%; leukopenia, 22.5\%; peripheral sensory neuropathy, $9.7 \%$; febrile neutropenia (FN), 9.6\%; myalgia, 5.7\%; hepatobiliary disorders, 5.5\%; and arthralgia, $4.8 \%$ (Table 4 ). The AEs were different between $\mathrm{q} 3 \mathrm{w}$ and weekly nab-PTX. Neutropenia (19.9 vs. $48.0 \%, p<0.0001$ ), leukopenia (18.5 vs. $35.8 \%$, $p<0.0001$ ), peripheral sensory neuropathy (7.3 vs. $17.9 \%$, $p=0.0304)$, myalgia (3.3 vs. $14.0 \%, p<0.0001)$, arthralgia (2.3 vs. $13.5 \%, p<0.0001$ ), and vomiting ( 3.1 vs. $7.5 \%$, $p=0.0304$ ) were less frequent in the $\mathrm{q} 3 \mathrm{w}$ group than in the weekly group, respectively. However, hepatobiliary disorder was observed more frequently in the $\mathrm{q} 3 \mathrm{w}$ group than in the weekly group (6.4 vs. $2.3 \%, p=0.0304)$. Only one patient died of febrile neutropenia during the 5-fluorouracil, epirubicin, and cyclophosphamide (FEC) treatment.

\section{Dose of nab-PTX in drug sequence}

The total dose of nab-PTX administered to the patients is presented in Suppl. Table 3. The mean dose \pm standard deviation for all patients was $1060.6 \pm 237.9 \mathrm{mg}$. The total $\mathrm{q} 3 \mathrm{w}$ and weekly doses were $1004.3 \pm 116.7 \mathrm{mg}$ and $1263.9 \pm 358.0 \mathrm{mg}$, respectively $(p<0.0001)$. Regarding drug sequence, the total dose for anthracycline followed by nab-PTX was $1052.3 \pm 209.4 \mathrm{mg}$ (q3w: $1003.7 \pm 117.5 \mathrm{mg}$, weekly: $1372.4 \pm 353.0 \mathrm{mg} ; p<0.0001)$, and that for nabPTX followed by anthracycline was $1114.0 \pm 369.8 \mathrm{mg}$ (q3w: $1040 \mathrm{mg}$, weekly: $1159.0 \pm 332.3 \mathrm{mg} ; p=0.2875$ ).

\section{Prognosis for patients with pCR (ypTO/is ypN0) treated with nab-PTX-containing regimens}

The Kaplan-Meier curves of DFS and OS are shown in Fig. 3a. The DFS rates at 5 years were 80.7, 86.9, 90.0, and $75.5 \%$ for luminal, HER2-rich, luminal/HER2, and TNBC subtypes, respectively (Fig. 3a, left panel). The DFS rates stratified by pCR (ypT0/is ypN0) are shown in Fig. 3b (upper panel). In the HER2-rich and TNBC subtypes, the DFS for patients with pCR (ypT0/is ypN0) was significantly longer than that for patients without $\mathrm{pCR}$. The OS rates at 5 years were 89.6, 96.6, 97.1, and 77.4\% for luminal, HER2-rich, luminal/HER2, and TNBC subtypes, respectively (Fig. 3a, right panel). The OS rates stratified by pCR (ypT0/is ypN0) are shown in Fig. $3 b$ (lower panel). The OS for patients in TNBC was significantly longer with pCR than without pCR. However, in the luminal and luminal/HER2 subtypes, there was no statistical difference between the groups. The prognoses for pCR (ypT0 ypN0 and ypT0/is ypNX) were similar to those for pCR (ypT0/is ypN0) (Suppl. Fig. 4a, b).

\section{Discussion}

We analyzed the pCR rates using IPD data based on three pCR definitions [16]. Nodal involvement after NAC was associated with an increased risk of tumor recurrence and death, and a preferable prognosis was not associated with axillary residual tumors or intraductal tumors in the breast. Therefore, we recognize that ypT0 ypN0 and ypT0/ is ypN0 are clinically useful pCRs, particularly in patients with aggressive phenotypes such as HER2-rich or TNBC subtypes [22-24]. Our results indicated that the pCR rates of luminal-type tumors were $6.7,10.2$, and $13.4 \%$, respectively, which are similar to the findings for anthracycline and taxane chemotherapy, supporting the power of nab-PTX for ER-positive subtypes [25]. Although the multivariate analysis demonstrated that TNBC is statistically associated with nab-PTX-related pCR (OR: 2.97), the pCR rate (31.5\% for ypT0/is ypN0) was similar to previous findings for anthracycline and taxane chemotherapy [16, 22, 23]. However, recent studies have demonstrated that weekly nab-PTX administration induced a high pCR rate (41-49\%) [26-28]. In our study, the pCR rates for TNBC were $41.7 \%$ (20/48) by weekly nab-PTX and $28.8 \%$ (53/184) by q3w, respectively. The total dose of nab-PTX was higher with weekly administration than with q3w (Suppl. Table 3). Our findings might have been affected by the higher proportion of patients treated with q3w nab-PTX (83\%), resulting in a low pCR rate. Recent publications suggest new strategies, including dose-dense chemotherapy, platinum-containing regimens, or combinations with molecular-targeted agents lead to better results, showing $\geq 50 \%$ pCR rates [29-32]. It should be noted that TNBC-specific characteristics, including high NG, high Ki-67, and PD-L1 expression may have affected these results.

We hypothesized that nab-PTX would have a substantial effect on HER2-positive BC, and we subsequently found that the $\mathrm{pCR}$ (ypT0/is ypN0) rates were $63.5 \%$ for HER2-rich and $40.6 \%$ for luminal/HER2 subtypes. As reported in the NOAH trial and GeparQuattro study, the HER2-positive subtype showed good responses to anthracycline followed by taxane with trastuzumab, with pCR rates of 38-43.5\% [33, 34]. The pooled analysis indicated an additional power of $30-50 \%$ using trastuzumab [23]. In the NeoALTTO study, the pCR rate of the HER2-rich subtype reached $61.3 \%$ after treatment with paclitaxel and dual blockage using trastuzumab and lapatinib [35]. The NeoSphere trial also reported that the pCR rate was $63.2 \%$ after treatment with docetaxel combined with trastuzumab and pertuzumab [36]. In our study, the pCR rates reached 63.5\% (ypT0/is ypN0) for the HER2-rich subtype with nab-PTX and trastuzumab, and this was similar to 
a

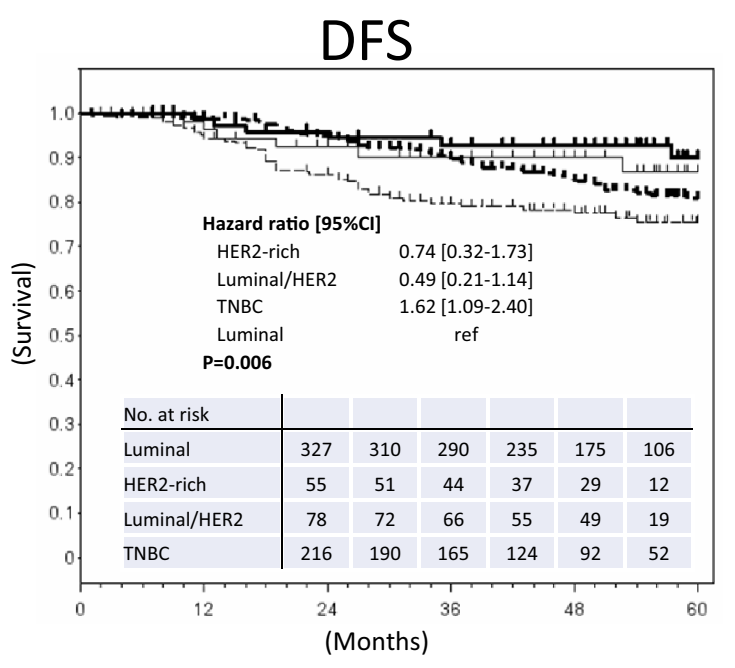

b

Luminal

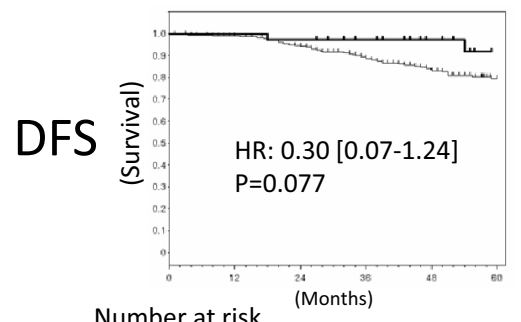

\begin{tabular}{c|c|c|c|c|c|c} 
Mo & 0 & 12 & 24 & 36 & 48 & 60 \\
\hline pCR(+) & 35 & 35 & 34 & 30 & 20 & 12 \\
\hline pCR(-) & 292 & 275 & 256 & 205 & 155 & 94
\end{tabular}

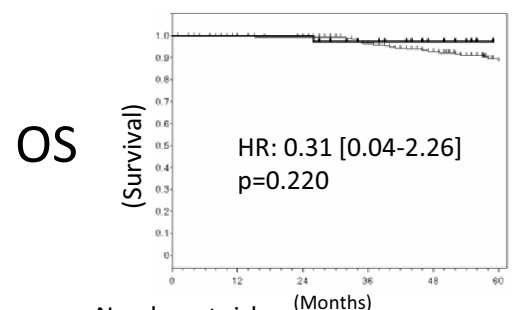

Number at risk (Months)

\begin{tabular}{c|c|c|c|c|c|c} 
Mo & 0 & 12 & 24 & 36 & 48 & 60 \\
\hline pCR(+) & 35 & 35 & 35 & 30 & 20 & 12 \\
\hline pCR(-) & 278 & 263 & 256 & 210 & 164 & 100
\end{tabular}

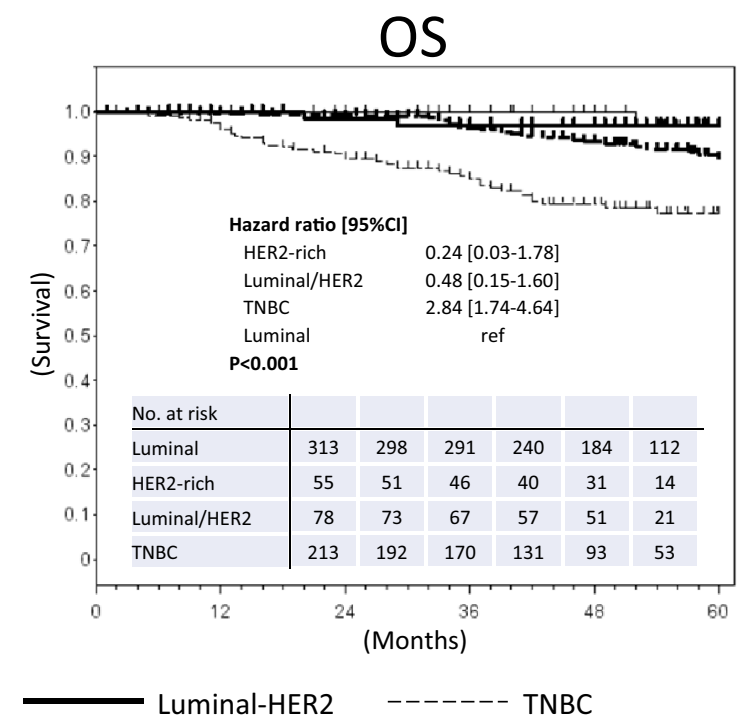

HER2-rich
UMminal-HER2
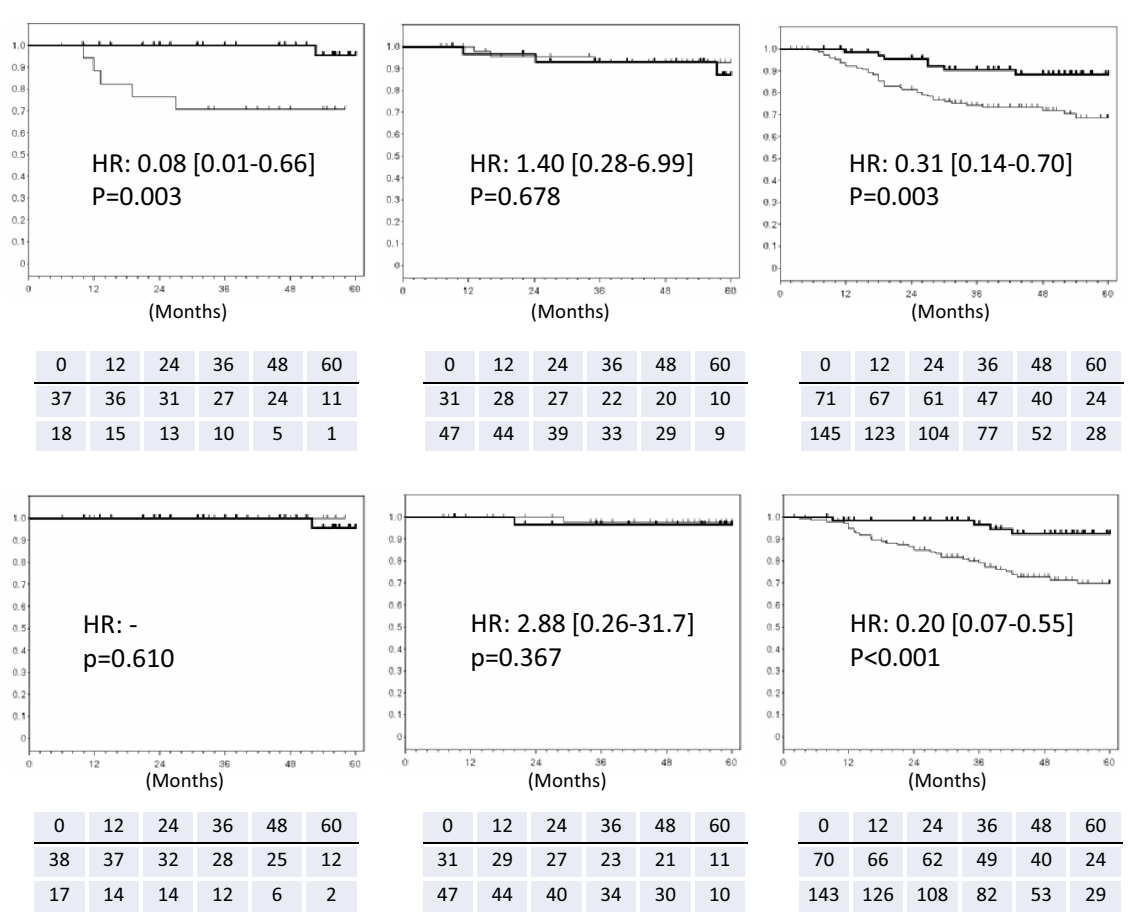

pCR
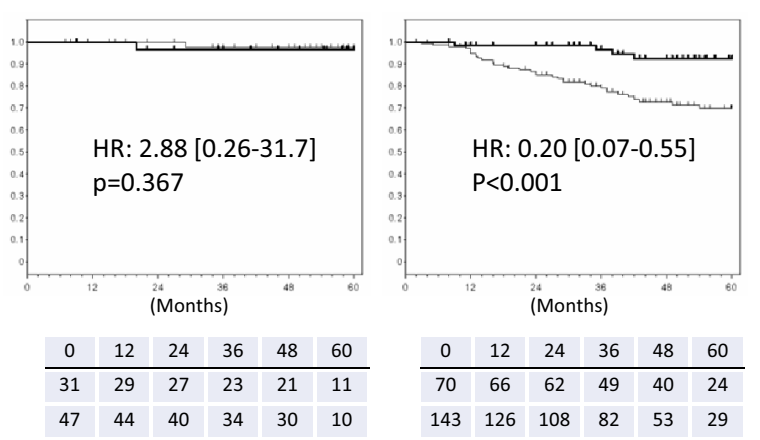

Fig. 3 Kaplan-Meier curves estimates for DFS and OS. a KaplanMeier estimates stratified by subtype. DFS (left) and OS (right) are shown with hazard ratios (HRs). b Kaplan-Meier estimates stratified by pCR (ypT0/is ypN0). Survival comparison between pCR and non-
PCR populations is indicated for DFS (upper panels) and OS (lower panels) in each subtype. The HR and $p$ value are indicated in each graph 
the value obtained with a dual anti-HER2 blockage regimen. In the GeparSepto trial, the combination of nab-PTX and dual blockage using pertuzumab and trastuzumab demonstrated remarkable results, showing that the pCR (ypT0 ypN0 and ypT0/is ypN0) rates reached 74.6 and $81.4 \%$, respectively, for the HER2-rich subtype [28]. We obtained results similar to those with the combination of docetaxel/paclitaxel and dual HER2 blockage described above. Despite no statistically significant difference, the pCR (ypT0/is ypN0) rates for the HER2-rich subtype were 59.7\% (q3w) and 76.5\% (weekly). The ORs for the HER2rich and luminal/HER2 subtypes in the multivariate analysis were 15.14 and 6.33 , respectively, which reflect the cases with high NG and/or Ki-67 expression, as shown in Table 2.

As shown in Table 4, our AE data were obtained using both nab-PTX and anthracycline. Hematological toxicities are reportedly common for both drugs [6, 26-28]. These results suggest that the toxicities of neoadjuvant nab-PTX are tolerable. Weekly nab-PTX produced more frequent and severe AEs than q3w nab-PTX for the following three reasons. (1) The total dose with weekly administration was considerably higher than that with $\mathrm{q} 3 \mathrm{w}$ regimen. (2) Weekly administration resulted in more frequent hospital visits to observe AEs than $\mathrm{q} 3 \mathrm{w}$ administration. (3) Our data were obtained from IPD with almost no missing data points, which enabled us to perform precise analyses.

Patients with pCR showed a better prognosis in all subtypes. In particular, the DFS in both HER2-rich and TNBC subtypes was longer with pCR than without pCR. Only TNBC patients with $\mathrm{pCR}$ were associated with an improved OS. Our study showed no difference in OS between $\mathrm{pCR}$ and non-PCR groups with the HER2-rich subtype, as observed in the NeoSphere study [37]. Although the NeoALLTO study showed a significant association between pCR and both DFS and OS, the OS of HER2-positive BC patients after NAC remains unclear because newly developed anti-HER2 therapies for metastatic BC may strongly affect long-term survival [38]. Our data demonstrated that nab-PTX for NAC induced higher pCR rate particularly in HER2-positive BC patients by combination with trastuzumab. These results may modify the adjuvant therapy against primary HER2positive BC. If pathologically negative lymph node is found after surgery, trastuzumab monotherapy may be enough in the adjuvant setting. However, in cases of positive lymph node or residual invasive disease, escalating therapies using either pertuzumab or trastuzumab emtansine (T-DM1) may be employed $[39,40]$. Patients with pCR who present long DFS could de-escalate the additional anti-HER2 therapies in the adjuvant setting and delay those in the metastatic setting. Although HER2-positive MBC may be sensitive to anti-HER2 therapy, it is the best drug for patients with primary breast cancer.
In this study, we assessed the risk of bias using RoBANS and classified it as moderate $[19,20]$. As most of the studies in this meta-analysis were not RCTs, the moderate risk overall does not indicate low study quality. The heterogeneity among the 16 studies was moderate $\left(I^{2}=68.8 \%\right)$. Unlike an RCT, the summary measure of a single-arm study directly reflects the distribution of the baseline characteristics. Actually, two studies showed low pCR rates in patients with luminal or TNBC subtypes, and high proportions of patients with cStage III. The existence of moderate heterogeneity indicates the need for IPD analysis to minimize heterogeneity.

In conclusion, nab-PTX is an acceptable chemotherapeutic agent for aggressive breast cancers such as HER2-rich, luminal/HER2, and TNBC subtypes in a neoadjuvant setting. Nab-PTX monotherapy is a useful option for TNBC. The combination of nab-PTX and anti-HER2 can achieve desirable pCR rates in patients with HER2-positive subtypes and manageable toxicity. Antibody drug conjugates, such as T-DM1 and trastuzumab deruxtecan (T-Dxd), are being developed in this field. Furthermore, trastuzumab and pertuzumab combined with taxane, and anti-microtubule agents are still considered standard preoperative or adjuvant therapy. Although there is a need for further clinical studies to replace the conventional docetaxel or paclitaxel with nabPTX, nab-PTX will be considered as a potential chemotherapeutic agent in combination with anti-HER2 antibodies to enhance their efficacy.

Supplementary Information The online version contains supplementary material available at https://doi.org/10.1007/s12282-021-01238-9.

Acknowledgments We thank all patients who participated in the studies. We appreciate the Japan Breast Cancer Research Group (JBCRG) administrative office for its contribution to the management of this study.

Author contributions All authors contributed to the study conception and design. Material preparation, data collection and analysis were performed by MF, MO and NM. The first draft of the manuscript was written by MF and MO, and all authors commented on previous versions of the manuscript. All authors read and approved the final manuscript.

Funding This work was funded by the Japan Breast Cancer Research Group (JBCRG). The research fund was provided to JBCRG by Taiho Pharmaceutical Co., Ltd. under the study contract.

\section{Declarations}

Conflict of interest MF-Remuneration: Chugai, Taiho, Takeda, Novartis, and Eisai. NM-Remuneration: Chugai, AstraZeneca, Pfizer, Eli-Lilly, Eisai, Takeda, Kyowa-Kirin, MSD, Novartis, NipponKayaku, and Daiichi-Sankyo. Funding: Chugai. HB-Remuneration: AstraZeneca, Eisai, Kyowa-Kirin, Taiho, Chugai, Nippon-Kayaku, Pfizer, and Novartis. YY-Remuneration and Funds: Novartis, NipponKayaku, Kyowa-Kirin, Taiho, and Chugai. TS-Remuneration: Ono, Kyowa-Kirin. Funding: Taiho, AstraZeneca, Chugai, and Eisai. UTRemuneration: Chugai, Eisai, and Kyowa-Kirin. Grant: Chugai, Eisai, 
Taiho, Daichi-Sankyo, Nippon-Kayaku, and Kyowa-Kirin. YK-Remuneration: Chugai, Novartis, Daiichi-Sankyo, Pfizer, Eli-Lilly, AstraZeneca, Kyowa-Kirin, and Eisai. TN-Remuneration: Chugai, Eli Lilly, Novartis, AstraZeneca, and Daiichi-Sankyo. SO-Remuneration: Chugai, Eisai, Eli-Lilly, Pfizer, Taiho, and AstraZeneca. Funding: Taiho and Eisai.

Ethical approval This study was conducted in accordance with the Declaration of Helsinki and approved by the review board or ethical committee of each participating institute.

Informed consent This meta-analysis used only existing information as described in the "Ethical Guidelines for Epidemiology Research", co-published by the Ministry of Education, Culture, Sports, Science and Technology and the Ministry of Health, Labour, and Welfare of Japan. The study purpose, methods, collection, and usage of data were published on a poster in each institution relevant to each subject population to give the opportunity to opt-out. Thus, written informed consent was not necessary.

Open Access This article is licensed under a Creative Commons Attribution 4.0 International License, which permits use, sharing, adaptation, distribution and reproduction in any medium or format, as long as you give appropriate credit to the original author(s) and the source, provide a link to the Creative Commons licence, and indicate if changes were made. The images or other third party material in this article are included in the article's Creative Commons licence, unless indicated otherwise in a credit line to the material. If material is not included in the article's Creative Commons licence and your intended use is not permitted by statutory regulation or exceeds the permitted use, you will need to obtain permission directly from the copyright holder. To view a copy of this licence, visit http://creativecommons.org/licenses/by/4.0/.

\section{References}

1. Ibrahim NK, Desai N, Legha S, Soon-Shiong P, Theriault RL, Rivera E, et al. Phase I and pharmacokinetic study of ABI-007, a cremophor-free, protein-stabilized, nanoparticle formulation of paclitaxel. Clin Cancer Res. 2002;8:1038-44.

2. Sparreboom A, Scripture CD, Trieu V, Williams PJ, De T, Yang $\mathrm{A}$, et al. Comparative preclinical and clinical pharmacokinetics of a cremophor-free, nanoparticle albumin-bound paclitaxel (ABI007) and paclitaxel formulated in cremophor (Taxol). Clin Cancer Res. 2005; 11:4136-43.

3. Desai N, Trieu V, Yao Z, Louie L, Ci S, Yang A, et al. Increased antitumor activity, intratumor paclitaxel concentrations, and endothelial cell transport of cremophor-free, albumin-bound paclitaxel, ABI-007, compared with cremophor-based paclitaxel. Clin Cancer Res. 2006;12:1317-24.

4. Gradishar WJ, Tjulandin S, Davidson N, Shaw H, Desai N, Bhar $\mathrm{P}$, et al. Phase III trial of nanoparticle albumin-bound paclitaxel compared with polyethylated castor oil-based paclitaxel in women with breast cancer. J Clin Oncol. 2005;23:7794-803.

5. Gradishar WJ, Krasnojon D, Cheporov S, Makhson AN, Manikhas GM, Clawson A, et al. Significantly longer progressionfree survival with nab-paclitaxel compared with docetaxel as first-line therapy for metastatic breast cancer. J Clin Oncol. 2009;27:3611-9.

6. Untch M, Jackisch C, Schneeweiss A, Conrad B, Aktas B, Denkert C, et al. Nab-paclitaxel versus solvent-based paclitaxel in neoadjuvant chemotherapy for early breast cancer (Gepar- Septo-GBG 69): a randomised, phase 3 trial. Lancet Oncol. 2016;17:345-56.
7. The University Hospital Medical Information Network Clinical Trials Registry (UMIN-CTR). https://www.umin.ac.jp/ctr/index-j. htm. Accessed 22 Aug 2017.

8. Futamura M, Nagao Y, Ishihara K, Takeuchi M, Nakada T, Kawaguchi Y, et al. Preoperative neoadjuvant chemotherapy using nanoparticle albumin-bound paclitaxel followed by epirubicin and cyclophosphamide for operable breast cancer: a multicenter phase II trial. Breast Cancer. 2017;24:615-23.

9. Shigematsu H, Kadoya T, Masumoto N, Sasada T, Emi A, Ohara $\mathrm{M}$, et al. The efficacy and safety of preoperative chemotherapy with triweekly abraxane and cyclophosphamide followed by 5-fluorouracil, epirubicin, and cyclophosphamide therapy for resectable breast cancer: a multicenter clinical trial. Clin Breast Cancer. 2015;15:110-6.

10. Shimada H, Ueda S, Saeki T, Shigekawa T, Takeuchi H, Hirokawa E, et al. Neoadjuvant triweekly nanoparticle albumin bound paclitaxel followed by epirubicin and cyclophosphamide for stage II/ III HER2-negative breast cancer: evaluation of efficacy and safety. Jpn J Clin Oncol. 2015;45:642-9.

11. Kuwayama T, Nakamura S, Hayashi N, Takano T, Tsugawa K, Sato T, et al. Randomized multicenter phase II trial of neoadjuvant therapy comparing weekly nab-paclitaxel followed by FEC with docetaxel followed by FEC in HER2 early-stage breast cancer. Clin Breast Cancer. 2018;18:474-80.

12. Kojima Y, Kawamoto H, Nishikawa T, Hayami R, Shimo A, Haku E, et al. Feasibility study of weekly nanoparticle albumin-bound paclitaxel $(150 \mathrm{mg} / \mathrm{m} 2)$ followed by fluorouracil, epirubicin, and cyclophosphamide therapy as neoadjuvant chemotherapy for HER2-negative breast cancer. Clin Breast Cancer. 2018;18:374-9.

13. Ishigami E, Sakakibara M, Sakakibara J, Iwase T, Hayama S, Masuda T, et al. Phase II study of neoadjuvant anthracycline combined with nanoparticle albumin bound paclitaxel for human epidermal growth factor receptor 2 negative breast cancer. Mol Clin Oncol. 2017;7:1079-82.

14. Stewart LA, Tierney JF. To IPD or not to IPD? Advantages and disadvantages of systematic reviews using individual patient data. Eval Health Prof. 2002;25:76-97.

15. Japan Breast Cancer Society. General rules for clinical and pathological study of breast cancer in Japan (17th edition). Tokyo: Kanehara Shuppan; 2012.

16. von Minckwitz G, Untch M, Blohmer JU, Costa SD, Eidtmann H, Fasching PA, et al. Definition and impact of pathologic complete response on prognosis after neoadjuvant chemotherapy in various intrinsic breast cancer subtypes. J Clin Oncol. 2012;30:1796-804.

17. Kim SY, Park JE, Lee YJ, Seo HJ, Sheen SS, Hahn S, et al. Testing a tool for assessing the risk of bias for nonrandomized studies showed moderate reliability and promising validity. J Clin Epidemiol. 2013;66:408-14.

18. Sterne JA, Hernán MA, McAleenan A, Reeves BC, Higgins JP. Chapter 25: Assessing risk of bias in a non-randomized study. Cochrane Training. 2019. https://training.cochrane.org/handbook/ current/chapter-25

19. Stewart LA, Clarke M, Rovers M, Riley RD, Simmonds M, Stewart $\mathrm{G}$, et al. Preferred reporting items for systematic review and meta-analyses of individual participant data: the PRISMA-IPD statement. JAMA. 2015;313:1657-65.

20. Eisenhauer EA, Therasse P, Bogaerts J, Schwartz LH, Sargent D, Ford R, et al. New response evaluation criteria in solid tumours: revised RECIST guideline (version 1.1). Eur J Cancer 2009;45:228-47.

21. Kuroi K, Toi M, Tsuda H, Kurosumi M, Akiyama F. Issues in the assessment of the pathologic effect of primary systemic therapy for breast cancer. Breast Cancer. 2006;13:38-48.

22. Guidance for Industry. Pathological complete response in neoadjuvant treatment of high-risk early-stage breast cancer: use as an endpoint to support accelerated approval. FDA guidance 
document https://www.fda.gov/regulatory-information/searchfda-guidance-documents/pathologic-complete-response-neoad juvant-treatment-high-risk-early-stage-breast-cancer-use-endpo int. Accessed 6 Mar 2020.

23. Cortazar P, Zhang L, Untch M, Mehta K, Costantino JP, Wolmark $\mathrm{N}$, et al. Pathological complete response and long-term clinical benefit in breast cancer: the $\mathrm{CTNeoBC}$ pooled analysis. Lancet. 2014;384:164-72.

24. Spring LM, Fell G, Arfe A, Sharma C, Greenup R, Reynolds $\mathrm{KL}$, et al. Pathologic complete response after neoadjuvant chemotherapy and impact on breast cancer recurrence and survival: a comprehensive meta-analysis. Clin Cancer Res. 2020;26:2838-48.

25. Furlanetto J, Jackisch C, Untch M, Schneeweiss A, Schmatloch $\mathrm{S}$, Aktas B, et al. Efficacy and safety of nab-paclitaxel $125 \mathrm{mg} / \mathrm{m}$ 2 and nab-paclitaxel $150 \mathrm{mg} / \mathrm{m} 2$ compared to paclitaxel in early high-risk breast cancer. Results from the neoadjuvant randomized GeparSepto study (GBG 69). Breast Cancer Res Treat. 2017;163:495-506.

26. Gianni L, Mansutti M, Anton A, Calvo L, Bisagni G, Bermejo B, et al. Comparing neoadjuvant nab-paclitaxel vs paclitaxel both followed by anthracycline regimens in women with ERBB2/HER2negative breast cancer. The evaluating treatment with neoadjuvant Abraxane (ETNA) trial. A randomized phase 3 clinical trial. JAMA Oncol. 2018;4:302-8.

27. Harbeck N, Zhang H, Barrios CH, Saji S, Jung KH, Hegg R, et al. Impassion031: results from a phase III study of neoadjuvant (neoadj) atezolismab + chemotherapy in early triple-negative breast cancer (TNBC). Ann Oncol. 2020;31:S1142-215.

28. Loibl S, Jackisch C, Schneeweiss A, Schmatloch S, Aktas B, Denkert C, et al. Dual HER2-blockade with pertuzumab and trastuzumab in HER2-positive early breast cancer: a subanalysis of data from the randomized phase III GeparSepto trial. Ann Oncol. 2017;28:497-504.

29. Schneeweiss A, Möbus V, Tesch H, Hanusch C, Denkert C, Lübbe $\mathrm{K}$, et al. Intense dose-dense epirubicin, paclitaxel, cyclophosphamide versus weekly paclitaxel, liposomal doxorubicin (plus carboplatin in triple-negative breast cancer) for neoadjuvant treatment of high-risk early breast cancer (GeparOcto-GBG 84): A randomised phase III trial. Eur J Cancer. 2019;106:181-92.

30. von Minckwitz G, Schneeweiss A, Loibl S, Salat C, Denkert C, Rezai M, et al. Neoadjuvant carboplatin in patients with triple-negative and HER2-positive early breast cancer (GeparSixto; GBG 66): a randomised phase 2 trial. Lancet Oncol. 2014;15:747-56.

31. Loibl S, O'Shaughnessy J, Untch M, Sikov WM, Rugo HS, McKee MD, et al. Addition of the PARP inhibitor veliparib plus carboplatin or carboplatin alone to standard neoadjuvant chemotherapy in triple-negative breast cancer (BrighTNess): a randomised, phase 3 trial. Lancet Oncol. 2014;19:497-509.

32. Loibl S, Untch M, Burchardi N, Huober JB, Blohmer JU, Grischke E-M, et al. Randomized phase II neoadjuvant study (GeparNuevo) to investigate the addition of durvalumab to a taxane-anthracycline containing chemotherapy in triple negative breast cancer (TNBC). J Clin Oncol. 2018;36:104.

33. Gianni L, Eiermann W, Semiglazov V, Manikhas A, Lluch A, Tjulandin S, et al. Neoadjuvant chemotherapy with trastuzumab followed by adjuvant trastuzumab versus neoadjuvant chemotherapy alone, in patients with HER2-positive locally advanced breast cancer (the NOAH trial): a randomised controlled superiority trial with a parallel HER2-negative cohort. Lancet. 2010;375:377-84.

34. Untch M, Rezai M, Loibl S, Fasching PA, Huober J, Tesch H, et al. Neoadjuvant treatment with trastuzumab in HER2-positive breast cancer: results from the GeparQuattro study. J Clin Oncol. 2010;28:2024-31.

35. Baselga J, Bradbury I, Eidtmann H, Di Cosimo S, de Azambuja E, Aura C, et al. Lapatinib with trastuzumab for HER2-positive early breast cancer (NeoALTTO): a randomised, open-label, multicentre, phase 3 trial. Lancet. 2012;379:633-40.

36. Gianni L, Pienkowski T, Im YH, Roman L, Tseng LM, Liu MC, et al. Efficacy and safety of neoadjuvant pertuzumab and trastuzumab in women with locally advanced, inflammatory, or early HER2-positive breast cancer (NeoSphere): a randomised multicentre, open-label, phase 2 trial. Lancet Oncol. 2012;13:25-32.

37. Gianni L, Pienkowski T, Im YH, Tseng LM, Liu MC, Lluch A, et al. 5-year analysis of neoadjuvant pertuzumab and trastuzumab in patients with locally advanced, inflammatory, or early-stage HER2-positive breast cancer (NeoSphere): a multicentre, openlabel, phase 2 randomised trial. Lancet Oncol. 2015;17:791-800.

38. Huober J, Holmes E, Baselga J, de Azambuja E, Untch M, Fumagalli D, et al. Survival outcomes of the NeoALTTO study (BIG 1e06): updated results of a randomised multicenter phase III neoadjuvant clinical trial in patients with HER2-positive primary breast cancer. Eur J Cancer. 2019;118:169-77.

39. von Minckwitz G, Procter M, Azambuja E, Zardavas D, Benyunes $\mathrm{M}$, Viale $\mathrm{G}$, et al. Adjuvant pertuzumab and trastuzumab in early HER2-positive breast cancer. N Engl J Med. 2017;377:122-31.

40. von Minckwitz G, Huang CS, Mano MS, Loibl S, Mamounas EP, Untch M, et al. Trastuzumab emtansine for residual invasive HER2-positive breast cancer. N Engl J Med. 2019;380:617-28.

Publisher's Note Springer Nature remains neutral with regard to jurisdictional claims in published maps and institutional affiliations.

\section{Authors and Affiliations}

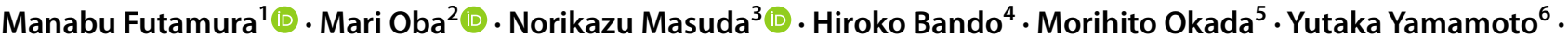 Takanori Kin ${ }^{7}$. Toshiaki Saeki ${ }^{8}$ Takeshi Nagashima ${ }^{9} \cdot$ Takashi Kuwayama $^{10}$. Uhi Toh ${ }^{11}$ • Akira Hirano ${ }^{12}$. Masafumi Inokuchi ${ }^{13} \cdot$ Kazuhiko Yamagami $^{14} \cdot$ Yutaka Mizuno $^{15} \cdot$ Yasuyuki Kojima $^{16}$ (1) Takahiro Nakayama $^{17}$. Hiroyuki Yasojima ${ }^{3} \cdot$ Shinji Ohno ${ }^{18}$}

1 Department of Surgical Oncology, Gifu University Graduate School of Medicine, Gifu 501-1194, Japan

2 Department of Medical Statistics, Toho University, Tokyo 143-8540, Japan
3 Department of Surgery, Breast Oncology, National Hospital Organization Osaka National Hospital, Osaka 540-0006, Japan

4 Department of Breast-Thyroid-Endocrine Surgery, Tsukuba University, Tsukuba 305-8576, Japan 
5 Research Institute for Radiation Biology and Medicine, Hiroshima University, Hiroshima 734-8553, Japan

6 Department of Breast and Endocrine Surgery, Graduate School of Medical Sciences, Kumamoto University, Kumamoto 860-8556, Japan

7 Department of Breast Surgery, Hiroshima City Hiroshima Citizens Hospital, Hiroshima 730-8518, Japan

8 Department of Breast Oncology, Saitama Medical University International Medical Center, Saitama 350-1298, Japan

9 Department of General Surgery, Graduate School of Medicine, Chiba University, Chiba 260-8677, Japan

10 Division of Breast Surgical Oncology, Showa University, Tokyo 142-8666, Japan

11 Department of Surgery, Kurume University School of Medicine, Kurume 830-0011, Japan
12 Department of Breast Surgery, Medical Center East, Tokyo Women's Medical University, Tokyo 116-8567, Japan

13 Department of Breast and Endocrine Surgery, Kanazawa Medical University, Ishikawa 920-0293, Japan

14 Department of Breast Surgery and Oncology, Shinko Hospital, Kobe 651-0072, Japan

15 Department of Breast Surgery, Yokkaichi Municipal Hospital, Yokkaichi 510-8567, Japan

16 Division of Breast and Endocrine Surgery, Department of Surgery, St. Marianna University School of Medicine, Kawasaki 216-8511, Japan

17 Department of Breast and Endocrine Surgery, Osaka International Cancer Institute, Osaka 541-8567, Japan

18 Breast Oncology Center, Cancer Institute Hospital, Tokyo 135-0063, Japan 